\title{
Explorative study to identify novel candidate genes related to oxaliplatin efficacy and toxicity using a DNA repair array
}

\author{
DM Kweekel, ${ }^{*,}$, NF Antonini ${ }^{2}$, JWR Nortier ${ }^{3}$, CJA Punt ${ }^{4}$, H Gelderblom ${ }^{3}$ and H-J Guchelaar' \\ 'Department of Clinical Pharmacy and Toxicology, Leiden University Medical Center, Leiden, The Netherlands; ${ }^{2}$ Biometrics Department, Netherlands \\ Cancer Institute (NKI), Amsterdam, The Netherlands; ${ }^{3}$ Department of Clinical Oncology, Leiden University Medical Center, Leiden, The Netherlands; \\ ${ }^{4}$ Department of Oncology, Radboud University Nijmegen Medical Center, Nijmegen, The Netherlands
}

PURPOSE: To identify new polymorphisms (single nucleotide polymorphisms, SNPs) in DNA repair pathways that are associated with efficacy and toxicity in patients receiving oxaliplatin and capecitabine for advanced colorectal cancer (ACC).

METHODS: We studied progression-free survival (PFS) in 91 ACC patients, of whom germ-line DNA was isolated and genotyped using an Asper Biotech array. Overall survival (OS) and toxicity were studied as secondary end points. A step-wise selection of SNPs was performed, involving univariate and multivariate log-rank tests and Cox regression analysis, with age and performance status as covariates. RESULTS: A total of 8 I SNPs in 46 genes on the array were selected for further analysis, based on genotyping success rates and minor allele frequencies. After step-wise selection, we found that homozygosity for the ataxia telangiectasia mutated gene (ATM) rs/80।5I6 or excision repair cross-complementing gene (ERCC5) rs 047768 SNPs was associated with shorter PFS; however there were no significant associations $(P>0.01)$ with OS or toxicity.

DISCUSSION: This is the first study describing the pathway gene approach for the selection of new candidate genes involved in oxaliplatin efficacy and toxicity. The results suggest that the ATM and ERCC5 genes may be associated with oxaliplatin efficacy in ACC.

British Journal of Cancer (2009) I OI, 357-362. doi:10.1038/sj.bjc.6605 I 34 www.bjcancer.com

Published online 16 June 2009

(c) 2009 Cancer Research UK

Keywords: SNP array; oxaliplatin; efficacy; toxicity; ATM; ERCC5

Oxaliplatin is a cytotoxic anti-tumour agent that is frequently used in advanced colorectal cancer (ACC; Punt, 2004). Its use results in platination of (tumour) DNA and the formation of platinum/DNA cross-links. Oxaliplatin adducts are lethal to cells, and may be removed from the DNA through a number of DNA repair pathways. Polymorphisms in the nucleotide excision repair (NER), base excision repair (BER) and mismatch repair (MMR) pathways were found to be associated with DNA repair after oxaliplatin exposure (reviewed by Kweekel et al, 2005).

The classical way of studying associations between drug effects and genetic variation is by the candidate gene method. This method involves the careful selection of single nucleotide polymorphisms (SNPs) based on the functionality of the genetic variant. However, replication of associations as described in the literature has not always been possible. For a large extent, this is due to the fact that most drug effects are considered as complex traits and are therefore not causally linked to one single gene or SNP. An alternative to the candidate gene method is the wholegenome approach (WGA). The main advantage of this method is that it is hypothesis free because it does not rely on current understanding of the pharmacokinetics and -dynamics of a drug. However, false-positive results that occur from multiple testing are a major concern in this type of research. The pathway gene method is a combination of both methods; instead of candidate genes, candidate pathways are selected based on the pharmacodynamic and pharmacokinetic behaviour of a drug. After selecting the appropriate pathways, SNPs in the corresponding genes are

*Correspondence: Dr DM Kweekel; E-mail: d.m.kweekel@lumc.nl Received 5 February 2009; revised 14 May 2009; accepted 21 May 2009; published online 16 June 2009 included in association analysis. Compared to the WGA, the pathway method involves a limited number of statistical association tests while at the same time allowing a broader part of the genome to be tested compared to the candidate gene method. The risk of false-positive findings is lower compared to WGA; also, because more enzymes in a pathway are studied, finding an association with the most relevant gene is more likely (Wang et al, 2007). Asper Biotech offers DNA repair chips that determine 100 SNPs in 56 genes, including SNPs in the homologous repair pathway, as well as SNPs in the BER, NER and MMR pathways. Moreover, it bears SNPs from several genes related to the control of cell-cycle and apoptosis pathways (including cyclin-dependent kinases p16 and p21, and tumour suppressor protein p53; http:// www.asperbio.com/DNArepair.htm). These DNA repair chips are promising means of investigating associations with response or toxicity in patients receiving platinum derivatives, using the pathway gene approach.

The purpose of the current study was to perform an explorative association study of DNA repair pathway SNPs with progressionfree survival (PFS) in ACC patients receiving with oxaliplatin combination therapy. Secondary end points were overall survival (OS) and toxicity.

\section{MATERIALS AND METHODS}

\section{Patients}

Germ-line DNA was obtained from peripheral blood of Caucasian patients with ACC who participated in the Dutch CAIRO trial. The inclusion criteria and the clinical results of this study have been 
published elsewhere (Koopman et al, 2006). Patients were included in the current side study if they were randomised to arm B (combinational chemotherapy, starting with first-line irinotecan plus capecitabine) and continued to second-line therapy, which consisted of oxaliplatin $\left(130 \mathrm{mg} \mathrm{m}^{-2}\right.$ on day 1$)$ and capecitabine $\left(1000 \mathrm{mg} \mathrm{m}^{-2}\right.$, b.i.d. on days $\left.1-14\right)$, every 3 weeks until progression or unacceptable toxicity. Dose reductions were performed for capecitabine in case of grades $2-4$ toxicity as described previously (Van Cutsem et al, 2001). Oxaliplatin dose reductions of $25 \%$ were carried out in case of grade 4 haematological toxicity, febrile neutropenia and for persistent paresthesias $(\geqslant 14$ days, grade 1 neurotoxicity) or temporary (7-14 days) painful paresthesias/ functional impairment (grades $\geqslant 2$ neurotoxicity). Patients experiencing persistent grades $\geqslant 2$ neurotoxicity received a $50 \%$ oxaliplatin dose reduction. If haematological and non-haematological toxicities had not recovered to grade 1 before the next treatment cycle, oxaliplatin dose was delayed for a maximum of 2 weeks. If still not recovered by that time, patients went off-study. Prophylactic use of haematological growth factors was not permitted. The accrual period was from January 2003 to December 2004, and EDTA blood samples for genotyping were collected from December 2003 to March 2005 after a protocol amendment. The objective of this amendment was to perform genetic association studies regarding drug efficacy and toxicity. The study protocol and the amendment were approved by the local ethics committees. Written informed consent was obtained from all patients participating in the genetic association study before blood collection. Tumour evaluation was performed every three cycles according to RECIST criteria (Therasse et al, 2000) and toxicity was graded according to US National Cancer Institute Common Toxicity Criteria, version 2.0. The primary end point of this study was association of individual SNPs with PFS. Secondary end points were associations with OS and the incidence of overall worst grades 3-4 toxicity. Progression-free survival was calculated as the time from the start of second-line treatment with oxaliplatin to progression, death or loss to follow-up, whichever came first. Overall survival was also calculated as the time from the start of second-line oxaliplatin treatment to death or loss to follow-up. Progression-free survival was preferred as primary end point over OS, because it reflects oxaliplatin efficacy and is not potentially influenced by salvage therapies.

\section{Genotyping}

Peripheral EDTA blood was stored at $-20^{\circ} \mathrm{C}$ before isolation with the Magnapure LC (Roche Diagnostics, Almere, the Netherlands) according to the manufacturer's instructions. Asper Biotech (Tartu, Estonia) designed and performed a DNA repair chip (including 100 DNA repair SNPs in 55 genes) according to proprietary protocols. The chip contained SNPs of the following pathways: BER (XRCC1, XRCC2, XRCC3, XRCC4, XRCC5, XRCC9, APEX, POLB, LIG4, MYH, OGG1), NER (ERCC1, ERCC2, ERCC4, ERCC5, LIG1, LIG3, RAD23B, XPA, XPC), MMR (MLH1, MSH2, MSH3, MSH6, PMS2, RECQL), double-strand break repair and HR (homologous recombination: BARD1, BRCA1, BRCA2, FANCD2, NBS1, RAD51, RAD52, RAD54B, PARP1, PARP4), cell-cycle regulation (ATM, CCND1, CCNH,CDKN1B,CDKN2B,CDK7, CHEK2, RAD9A, TP53, TP53BP1, TP53BP2, p21, CDKN2A, GRTH, $G A D D 45 A)$ and other enzymes involved in DNA synthesis (CDA, NT5E, PCNA, MGMT).

As a quality control check, we compared the results of the Asper Biotech array with six SNPs that had previously been determined by our own laboratory in the same set of 91 DNA samples (Van der Straaten et al, 2006) and unpublished data (available on request). We found that, depending on the SNP, only $0.0-3.6 \%$ of the samples showed results that were discordant, which suggests good quality of the array and the procedures that were used in genotyping. All genotype data are available on request.

\section{Statistics}

Patients randomised to the combination treatment who started second-line oxaliplatin/capecitabine combination therapy and had SNP assessment were eligible for the analysis $(N=91)$. A SNP was considered evaluable for analysis if at least $90 \%$ of the patients were successfully genotyped, and if the minor allele frequency (MAF) was at least $5 \%$.

Log-rank tests and Cox proportional hazards regression were performed to investigate the association with PFS/OS. Logistic regression analysis was performed to investigate the association with toxicity. If more than three patients were homozygous for the mutant allele, we tested both the separate genotypes individually and the combination of genotypes using the dominant or recessive model (carrier analysis). If three or less patients were homozygous mutant, we only compared the wild-type patients with the mutant allele carriers.

The purpose of this study was to explore the possible associations of the clinical end points of each SNP, either as a separate factor or combined. We used a two-step approach. In the first step, we performed the univariate analysis of PFS, OS and overall worst grades 3-4 toxicity for each SNP separately. Single nucleotide polymorphisms with a $P$-value of $<0.01$ were considered statistically significant and selected for the next step. In the second step, a covariate analysis with age and a performance status of 2 at the start of therapy (according to WHO guidelines) was performed for each SNP separately. On the basis of previously published results (Koopman et al, 2007), both a WHO performance status of 2 and abnormal serum lactate dehydrogenase (LDH) should ideally have been selected as covariates. However, $\mathrm{LDH}$ was underreported in most patients at the start of second-line treatment and therefore not used in the analysis. Age was selected as a covariate because it generally associates with survival. Finally, SNPs with a $P$-value of $<0.01$ were analysed in combination, in one multivariate model with the same covariates as in the previous step. This last analysis was performed for exploratory purposes only, to evaluate the relative importance of the individual factors.

Correction for multiple testing was carried out. However, a Bonferroni correction (based on the number of SNPs) would be far too conservative in this case, because it assumes that the SNPs investigated are all independent. Clearly, many of these SNPs are not only in linkage disequilibrium but also the genes studied are part of interacting pathways. Therefore, the only rational option to correct for multiple testing is to account for the number of pathways tested (in this case BER, NER, MMR, HR and cell-cycle regulation, $n=5$ ). One could argue that correcting for the number of pathways even is too conservative, because these particular pathways are not independent entities either (Curtis et al, 2005). Moreover, a strict Bonferroni correction would negatively affect the explorative nature of the study and would increase the chance for false-negative results. For these reasons, we decided to set $\alpha$ at 0.01 . Throughout the paper, we present unadjusted $P$-values.

\section{RESULTS}

A total of 91 patients were eligible for the current analysis. DNA from these patients was genotyped using an SNP array with 100 SNPs on 55 genes. Two of these SNPs were excluded from analysis because of low genotyping success rates $(<90 \%)$. Seventeen SNPs were excluded because of MAF $<5 \%$. The remaining 81 SNPs in 46 genes were used for analysis of PFS, OS and grades 3-4 toxicity. All patients had genotype call rates exceeding $90 \%$.

\section{Step I: univariate analysis of oxaliplatin efficacy and toxicity}

Table 1 shows the SNPs for which the univariate analysis of PFS and OS yielded log-rank $P$-values $<0.05$ ( $P$ for overall log rank). 
Table I Step I: SNPs with $P<0.05$ in univariate analysis of PFS, OS and grades $3-4$ toxicity

PFS

ATM rs 1801516 (Asp 1853Asn)

Ataxia telangiectasia mutated gene

ERCC5 rs 1047768 (T335C, His46His)

$X P G$, excision repair cross-complementing (5) gene

GADD45A rs532446 (T3812C)

Growth arrest and DNA-damage-inducible gene, $\alpha$

RAD5 I rs 1801320 (G287C, Arg 96Pro)

RecA homologue gene

ATM rs609429 (IVS48+238 C > G)

Ataxia telangiectasia mutated gene

OGGI rs 1052133 (Ser 326Cys)

8-Oxoguanine DNA glycosylase gene

BRCA2 rs 15869 ( 105 bp 3'of STP A > C)

Breast cancer 2, early onset gene

OS

LIG4 rs|805388 (-176 C>T, Thr 9lle)

DNA ligase IV gene

BARD I rs2070093 (His 506His)

BRCA I-associated RING domain I gene

OGGI rs 1052133 (Ser 326Cys)

8-Oxoguanine DNA glycosylase gene

GADD45A rs532446 (T38I2C)

Growth arrest and DNA-damage-inducible gene, $\alpha$

ATM rs609429 (IVS48+238 C> G)

Ataxia telangiectasia mutated gene

LIG4 rs $1805389(-194$ C > T)

DNA ligase IV gene

MUTYH rs3219489 (Gln 324His)

MUT Y homologue

XRCCI rs25487 (GI30IA, Arg 399GIn)

$\mathrm{X}$-ray repair complementing defective repair (I)

Toxicity

ERCC2 rs238406 (C499A, Arg156Arg)

XPD (excision repair cross-complementing rodent

repair deficiency, complementation group 2)

MGMT rsI803965 (CI7IT, Leu 53Leu)

O-6-methylguanine-DNA methyltransferase

MGMT rs 12917 (C262T, Leu 84Phe)

O-6-methylguanine-DNA methyltransferase

LIGI rs3730849 (IVS2+12 C>T)

DNA ligase I
Regulator of tumour suppressor proteins p53 and BRCAI, checkpoint kinase CHK2, checkpoint proteins RADI7 and RAD9 and DNA repair protein NBSI.

Involved in excision repair of UV-induced DNA damage.

The protein product of this gene responds to environmental stresses by mediating activation of the p38/JNK pathway through MTKI/MEKK4 kinase.

Involved in the homologous recombination and repair of DNA.

0.016

Regulator of tumour suppressor proteins p53 and BRCAI, checkpoint kinase CHK2, checkpoint proteins RADI7 and RAD9, and DNA repair protein NBSI.

Excision of 8-oxoguanine, a mutagenic base by-product that occurs as a result of exposure to reactive oxygen.

Involved in maintenance of genome stability, specifically the homologous recombination pathway for double-strand DNA repair.

DNA ligase that joins single-strand breaks through non-homologous end joining. This protein forms a complex with the X-ray repair cross-complementing protein 4 (XRCC4).

Forms a complex with BRCAI that may be an essential aspect of tumour suppression by BRCAI.

0.009

Excision of 8-oxoguanine, a mutagenic base by-product that occurs as a result of exposure to reactive oxygen.

The protein product of this gene responds to environmental stresses by mediating activation of the p38/JNK pathway through MTKI/MEKK4 kinase.

Regulator of tumour suppressor proteins p53 and BRCAI, checkpoint kinase CHK2, checkpoint proteins RADI7 and RAD9, and DNA repair protein NBSI.

DNA ligase that joins single-strand breaks through non-homologous end joining. This protein forms a complex with the X-ray repair cross-complementing protein 4 (XRCC4),

Encodes a DNA glycosylase involved in oxidative DNA damage repair; excises adenine bases that are inappropriately paired with, eg 8-oxo-7,8-dihydroguanine (major oxidative DNA damage).

Involved in the efficient repair of DNA single-strand breaks formed by exposure to ionizing radiation and alkylating agents.

The protein encoded by this gene is involved in transcription-coupled nucleotide excision repair of damaged DNA, and is an integral member of the basal transcription factor BTF2/TFIIH complex.

DNA repair gene regulated by $\mathrm{p} 53$, confers resistance to alkylating agents

DNA repair gene regulated by $\mathrm{p} 53$, confers resistance to alkylating agents

LIGI encodes DNA ligase I, with functions in DNA replication and the base excision repair process. Mutations in LIGI that lead to DNA ligase I deficiency result in immunodeficiency and increased sensitivity to DNA-damaging agents.

SNPs with $P<0.01$ (in bold) were selected for further analysis. $* P$ is the overall log-rank $P$-value. Rs numbers and functions are derived from the NCBI Entrez SNP database, accessed December 2008 (http://www.ncbinlm.nih.gov/sites/entrez).

Among others, these include the genes ATM (ataxia telangiectasia mutated gene, two SNPs), OGG1 (8-oxoguanine DNA glycosylase gene), LIG4 (DNA ligase IV gene, two SNPs) and GADD45A (growth arrest and DNA-damage-inducible gene $\alpha$ ). The ATM, GADD45A and OGG1 SNPs show (a trend towards) association with both PFS and OS. On the basis of their $P$-values, we selected 
Table 2 Genotype distributions of SNPs, selected by univariate analysis of PFS, OS and toxicity

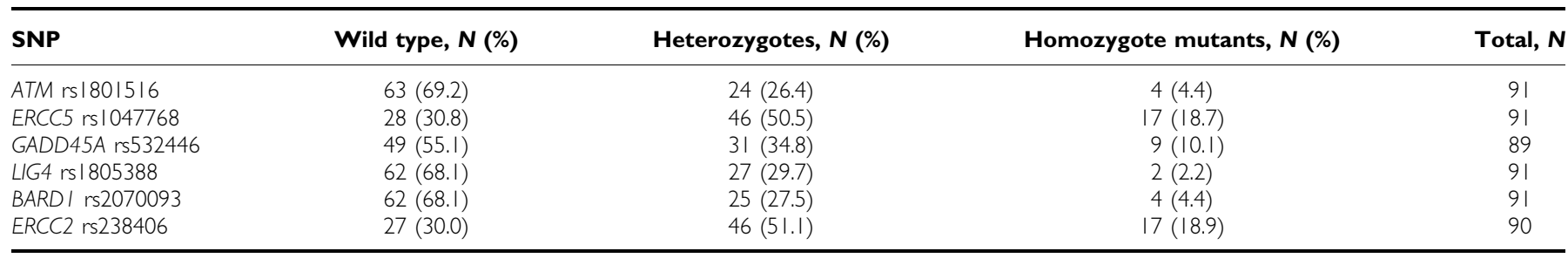

Table 3 Step II: hazard ratios (Cls) of treatment outcome for colorectal cancer patients receiving oxaliplatin, after adjusting for covariates

\begin{tabular}{|c|c|c|c|c|c|c|c|}
\hline \multirow[t]{3}{*}{ Progression-free survival } & ATM rs 1801516 & $0.72(0.43 ; 1.21)$ & $\begin{array}{c}4.25(1.45 ; 12.44) \\
\quad P=\mathbf{0 . 0 0 8}\end{array}$ & $\begin{array}{c}4.60(1.58 ; 3.37) \\
\boldsymbol{P}=\mathbf{0 . 0 0 5} *\end{array}$ & $0.98(0.96 ; 1.01)$ & $1.80(0.71 ; 4.55)$ & 0.009 \\
\hline & ERCC5 rs 1047768 & ।.7| (0.98; 2.98) & $\begin{array}{c}2.85(1.42 ; 5.7 \mid) \\
\boldsymbol{P}=\mathbf{0 . 0 0 3}\end{array}$ & $\begin{array}{c}1.92(1.13 ; 3.27) \\
P=0.016 * *\end{array}$ & $0.98(0.96 ; 1.00)$ & $1.62(0.63 ; 4.13)$ & 0.012 \\
\hline & GADD45A rs532446 & $1.58(0.95 ; 2.62)$ & I.I $4(0.53 ; 2.46)$ & $\begin{array}{c}1.44(1.04 ; 1.99) \\
P=0.029 * *\end{array}$ & $0.98(0.96 ; 1.00)$ & $1.56(0.60 ; 4.04)$ & 0.216 \\
\hline & BARD / rs2070093 & $\begin{array}{c}1.81(1.05 ; 3.12) \\
P=0.034\end{array}$ & $1.56(0.56 ; 4.38)$ & $\begin{array}{c}1.76(1.05 ; 2.95) \\
P=0.03 \mid * *\end{array}$ & $0.98(0.96 ; 1.01)$ & $1.38(0.44 ; 3.50)$ & 0.094 \\
\hline Toxicity grade $\geqslant 3$ & ERCC2 rs238406 & $\begin{array}{c}0.36(0.12 ; 1.09) \\
P=0.070\end{array}$ & $\begin{array}{c}0.10(0.02 ; 0.62) \\
\quad P=0.013\end{array}$ & $\begin{array}{c}0.28(0.10 ; 0.8 \mid) \\
P=0.0 \mid 8^{*} *\end{array}$ & $3.07(0.39 ; 24.29)$ & $\begin{array}{c}1.09(1.02 ; 1.17) \\
\boldsymbol{P}=\mathbf{0 . 0 0 8}\end{array}$ & 0.028 \\
\hline
\end{tabular}

\#P overall log rank P-value; carrier analysis: *P wild-type and heterozygotes vs homozygote mutants; **P heterozygotes plus homozygote mutants vs wild-type patients; performance status defined as stated in the Materials and methods section. All P-values in bold are significant according to the threshold set out in the Materials and methods section; other $P$-values are shown for clarification of confidence intervals only. In the carrier analysis, the most appropriate model (recessive or dominant) was chosen for combined analysis of genotypes, based on the individual HR of each category. Owing to the small number of LIG4 rs I 805388 homozygote mutant patients, no log rank $P$-value could be calculated to compare the 3 genotypes. SNPs with $P<0.01$ were selected for further analysis.

the ATM (rs1801516), ERCC5 (rs1047768) and GADD45A (rs532446) SNPs for further analysis of PFS; the LIG4 (rs1805388) and BARD1 (rs2070093) SNPs were selected for further analysis of OS.

With regard to overall toxicity grades $3-4$, the genes $O-6$ methylguanine-DNA methyltransferase (MGMT AGT, rs1803965 and rs12917) and ligase I (LIG1, rs3730849) showed $P$-values of $0.016,0.023$ and 0.031 respectively. ERCC2 (rs238406) was the only SNP significantly associated with grades $3-4$ toxicity $(P=0.007)$. Carriers of the mutant allele showed a lower risk of developing grades 3-4 toxicity. As only the ERCC2 SNP reached our criteria for significance, it was selected for further analysis.

\section{Step II: covariate analysis of oxaliplatin efficacy and toxicity}

We continued the analysis of SNPs selected in the first step by individual multivariate analysis, using age and $\mathrm{PS}=2$ as covariates. Genotype distributions of the selected SNPs are shown in Table 2. For OS, neither LIG4 rs1805388 $(P=0.017)$ nor BARD1 rs2070093 $(P=0.094)$ reached significance when corrected for the covariates. With regard to PFS however, we found that except for GADD45A (rs532446), the remaining ATM (rs1801516) and ERCC5 (rs1047768) SNPs were significantly associated (Table 3). Homozygote carriers of the ATM variant allele have a 4.25 times increased risk (confidence interval, CI: $1.45-12.44$ ) of progression on second-line combination chemotherapy of capecitabine plus oxaliplatin, compared to patients with the wild-type ATM $(P=0.008)$. Patients homozygous for the ERCC5 (excision repair cross-complementing gene, alternatively called $X P G$ ) variant allele also show an increased risk of progression compared to wild-type ERCC5 patients (HR for homozygote variant patients: 2.85; CI: $1.42-5.71, P=0.003$ ). Combined analysis of GADD45A (rs532446) with the covariates suggested no relevant association of this SNP with PFS (overall $P$ for log-rank analysis: 0.216 ). To obtain information on the relative impact on PFS of the ATM rs1801516 and ERCC5 rs1047768 SNPs, we performed a final multivariate analysis of both SNPs combined, corrected for performance status and age as covariates. We found that patients homozygous for the variant allele of $A T M$ rs 1801516 had a 3.2 times increased risk of progression (CI: $1.06-9.73, P=0.039$ ), whereas this risk was 2.4 times increased in patients homozygous for the ERCC5 rs 1047768 variant allele (CI: $1.14-4.99, P=0.021$ ).

With regard to toxicity, we found that the association of the $E R C C 2 \mathrm{rs} 238406$ SNP was diminished to $P=0.018$ after adjusting for the covariates. Patients carrying $\geqslant 1$ mutant ERCC2 allele have a 0.28 times risk to experience grades 3-4 toxicity during treatment with oxaliplatin (CI: $0.10-0.81$ ).

\section{DISCUSSION}

This is the first study describing the pathway gene approach for the selection of new candidate genes involved in oxaliplatin efficacy and toxicity. The results of this analysis suggest that ATM and ERCC5 might be involved in the efficacy of oxaliplatincontaining chemotherapy for ACC. The current report, although explorative in nature, may serve as a basis for further studies in other patient populations.

The ATM gene product interacts with the ERCC5 protein, and this complex prevents platinum-treatment-induced apoptosis (Colton et al, 2006). Two genetic variations in the ATM gene have already been studied in association with OS, including the intronic G60A (rs664143) and the T-77C (rs664677) SNP. Pancreatic cancer patients receiving gemcitabine and radiation with or without combined gemcitabine/cisplatin induction therapy have shorter OS 
if they carry the $-77 \mathrm{C}$ variant ( $\mathrm{Li}$ et al, 2006). A longer OS was reported in the same study population for patients who are homozygous for the 60A allele (Okazaki et al, 2008). The ATM SNP determined in the current study (rs1801516, or G5557A) has recently been shown to have an association with a reduced risk of breast cancer (Schrauder et al, 2008). This particular SNP is not in linkage with the ATM SNPs T-77C and G60A $\left(r^{2}=0.22\right.$, based on HapMap data phase II, March 2008). In the literature there are no data on the functionality of the Asp to Asn amino-acid substitution at this position. However, theoretically the substitution of the negatively charged Asp to neutral Asn may influence the ATM's interaction with other proteins. This may be a likely explanation, because the Asp amino acid at position 1853 appears to be highly conserved trough evolution (Schrauder et al, 2008).

The other variant, ERCC5 (rs1047768, or T335C), has been studied in ACC and ovarian cancer patients. A longer OS and PFS were reported for homozygous wild-type ACC patients treated with oxaliplatin/capecitabine combination therapy (Monzo et al, 2007), and similarly, a shorter OS was reported for homozygous mutant patients with ovarian cancer, treated with platinum-based chemotherapy (Saldivar et al, 2007). Although the results of the current study confirm these previous findings, it remains uncertain why a synonymous or 'silent' SNP would have clinical impact as the aminoacid sequence is unaltered. One explanation may be that this genetic variant is in linkage with other SNPs that do influence the amino-acid sequence of ERCC5. Alternatively, this silent ERCC5 SNP may result in a different protein conformation and hence different substrate specificity or enzyme activity, as was shown for several silent SNPs such as SNPs in the gene encoding P-glycoprotein (Komar, 2007).

Some of the SNPs detected in the presently used array have already been studied before by other groups, and we will summarise their findings for comparison. Previous studies included the SNPs ERCC1 rs11615 (C118T) and rs3212986 (C8092A), ERCC2 rs13181 (Lys751Gln) and rs238406 (C499A) as well as XRCC1 rs25487 (Arg399Gln). Besides the latter, none of these SNPs showed a (borderline) significant association with PFS or OS in the current univariate analysis. Various studies in colorectal cancer (Park et al, 2003; Stoehlmacher et al, 2004; Viguier et al, 2005; Ruzzo et al, 2007) and lung cancer patients (Su et al, 2007) suggest that the ERCC1 118CC genotype is associated with longer OS and a better response towards platinum agents. Another study reports no association of this SNP with OS (Zhou et al, 2004), but a significantly worse OS was found for patients carrying $\geqslant 1$ mutant allele of the other ERCC1 SNP, C8092A. For this SNP, an association with platinum-induced gastrointestinal toxicity was found by others (Suk et al, 2005). Regarding ERCC2, most of the research has focused on Lys751Gln. Some authors report no association of this SNP with clinical outcome (Giachino et al, 2007; Monzo et al, 2007) or toxicity (Le Morvan et al, 2007), whereas others describe a better anti-tumour response (Park et al, 2001; Ruzzo et al, 2007), longer OS (Stoehlmacher et al, 2004) and increased haematological toxicity (Booton et al, 2006) in wild-type homozygotes. For the other ERCC2 SNP, C499A, no association with OS or response has been reported (Park et al, 2001). Furthermore, the current study shows a trend towards longer OS in patients carrying $\geqslant 1$ mutant allele of XRCC1 Arg399Gln, which is in contrast to other studies reporting no association (Giachino et $a l, 2007)$ or a shorter OS in patients with a mutant allele (Gurubhagavatula et al, 2004).

A general problem of studies investigating a large set of SNPs is the risk of false-positive findings; for this reason we set our threshold for significance at $\alpha=0.01$. However, in this particular case, our major concern is false-negative results due to the relatively small sample size. This risk can only be minimised by using data from more patients, which, in turn, is not always feasible due to limited access to clinical data and patient samples. False-negative results due to low power can explain the inability to replicate the previously discussed associations found by other groups. However, the inability to replicate may also reflect differences in patient selection, publication bias, chance or low correlation of a marker with the outcome measure (Colhoun et al, 2003). Another concern of our study may be the low threshold that was set for the MAF ( $>5 \%)$. More specifically, it required that at least nine mutant alleles were genotyped for the given sample set. As a result, the power to detect associations with uncommon genetic variants was low. However, the commercial array included many SNPs with low MAFs (e.g. 17 SNPs were excluded because of MAF $<5 \%$ ). In our opinion, SNPs with low MAF should preferably not be included when developing an array.

In the palliative treatment of ACC, much progress has been made in recent years with the aid of pharmacogenetics. For instance, studies have revealed that patients with mutated $K-R A S$ oncogene do not benefit from cetuximab treatment (Karapetis et al, 2008). Febrile neutropenia, one of the side effects of irinotecan, can be predicted by the uridine diphosphate glucuronosyl transferase (UGT) $1 \mathrm{~A} 1{ }^{*} 28$ genotype (Kweekel et al, 2008). The results of the current study, although explorative in nature, need to be confirmed in a larger, independent cohort and may serve as a basis for new candidate SNP studies of genes located in the various DNA repair pathways.

\section{ACKNOWLEDGEMENTS}

The Dutch Colorectal Cancer Group (DCCG) CAIRO study was supported by the CKTO (grant 2002-07) and by unrestricted scientific grants from Roche, Sanofi-Aventis and Pfizer. We thank Tahar van der Straaten for his excellent technical assistance.

We thank the following CAIRO team members for participating in the pharmacogenetic side study: J van der Hoeven, Amstelveen; D Richel and B de Valk, Amsterdam; J Douma, Arnhem; P Nieboer, Assen; F Valster, Bergen op Zoom; G Ras and O Loosveld, Breda; D Kehrer, Capelle a/d IJssel; $M$ Bos, Delft; $H$ Sinnige and C Knibbeler, Den Bosch; W Van Deijk and H Sleeboom, Den Haag; E Muller, Doetinchem; E Balk, Ede; G Creemers, Eindhoven; R de Jong, Groningen; P Zoon, Harderwijk; J Wals, Heerlen; M Polee, Leeuwarden; M Tesselaar, Leiden; R Brouwer, Leidschendam; P de Jong and P Slee, Nieuwegein; H Oosten, Nijmegen; M Kuper, Oss; $M$ den Boer, Roermond; F de Jongh, Rotterdam; G Veldhuis, Sneek; D ten Bokkel Huinink, Utrecht; A van Bochove, Zaandam.

\section{Conflict of interest}

None of the authors report any relevant (financial) conflicts of interest.

\section{REFERENCES}

Booton R, Ward T, Heighway J, Taylor P, Power F, Ashcroft L, Morris J, Thatcher N (2006) Xeroderma pigmentosum group D haplotype predicts for response, survival, and toxicity after platinum-based chemotherapy in advanced nonsmall cell lung cancer. Cancer 106: 2421-2427

Colhoun HM, McKeigue PM, Davey SG (2003) Problems of reporting genetic associations with complex outcomes. Lancet 361: 865-872

Colton SL, Xu XS, Wang YA, Wang G (2006) The involvement of ataxiatelangiectasia mutated protein activation in nucleotide excision

repair-facilitated cell survival with cisplatin treatment. J Biol Chem 281: $27117-27125$

Curtis RK, Oresic M, Vidal-Puig A (2005) Pathways to the analysis of microarray data. Trends Biotechnol 23: 429-435

Giachino DF, Ghio P, Regazzoni S, Mandrile G, Novello S, Selvaggi G, Gregori D, DeMarchi M, Scagliotti GV (2007) Prospective assessment of XPD Lys751Gln and XRCC1 Arg399Gln single nucleotide polymorphisms in lung cancer. Clin Cancer Res 13: 2876-2881 
Gurubhagavatula S, Liu G, Park S, Zhou W, Su L, Wain JC, Lynch TJ, Neuberg DS, Christiani DC (2004) XPD and XRCC1 genetic polymorphisms are prognostic factors in advanced non-small-cell lung cancer patients treated with platinum chemotherapy. J Clin Oncol 22: 2594-2601

Karapetis CS, Khambata-Ford S, Jonker DJ, O'Callaghan CJ, Tu D, Tebbutt NC, Simes RJ, Chalchal H, Shapiro JD, Robitaille S, Price TJ, Shepherd L, $\mathrm{Au}$ HJ, Langer C, Moore MJ, Zalcberg JR (2008) K-ras mutations and benefit from cetuximab in advanced colorectal cancer. N Engl J Med 359: $1757-1765$

Komar AA (2007) Silent SNPs: impact on gene function and phenotype. Pharmacogenomics 8: $1075-1080$

Koopman M, Antonini NF, Douma J, Wals J, Honkoop AH, Erdkamp FL, de Jong RS, Rodenburg CJ, Vreugdenhil G, Akkermans-Vogelaar JM, Punt CJ (2006) Randomised study of sequential vs combination chemotherapy with capecitabine, irinotecan and oxaliplatin in advanced colorectal cancer, an interim safety analysis. A Dutch Colorectal Cancer Group (DCCG) phase III study. Ann Oncol 17: 1523-1528

Koopman M, Antonini NF, Douma J, Wals J, Honkoop AH, Erdkamp FL, de Jong RS, Rodenburg CJ, Vreugdenhil G, Loosveld OJ, van BA, Sinnige HA, Creemers GJ, Tesselaar ME, Slee PH, Werter MJ, Mol L, Dalesio O, Punt CJ (2007) Sequential vs combination chemotherapy with capecitabine, irinotecan, and oxaliplatin in advanced colorectal cancer (CAIRO): a phase III randomised controlled trial. Lancet 370: $135-142$

Kweekel DM, Gelderblom H, Guchelaar HJ (2005) Pharmacology of oxaliplatin and the use of pharmacogenomics to individualize therapy. Cancer Treat Rev 31: $90-105$

Kweekel DM, Gelderblom H, Van der Straaten T, Antonini NF, Punt CJ, Guchelaar HJ (2008) UGT1A1*28 genotype and irinotecan dosage in patients with metastatic colorectal cancer: a Dutch Colorectal Cancer Group study. Br J Cancer 99: 275-282

Le Morvan V, Smith D, Laurand A, Brouste V, Bellott R, Soubeyran I, Mathoulin-Pelissier S, Robert J (2007) Determination of ERCC2 Lys751Gln and GSTP1 Ile105Val gene polymorphisms in colorectal cancer patients: relationships with treatment outcome. Pharmacogenomics 8: $1693-1703$

Li D, Frazier M, Evans DB, Hess KR, Crane CH, Jiao L, Abbruzzese JL (2006) Single nucleotide polymorphisms of RecQ1, RAD54L, and ATM genes are associated with reduced survival of pancreatic cancer. J Clin Oncol 24: $1720-1728$

Monzo M, Moreno I, Navarro A, Ibeas R, Artells R, Gel B, Martinez F, Moreno J, Hernandez R, Navarro-Vigo M (2007) Single nucleotide polymorphisms in nucleotide excision repair genes $X P A, X P D, X P G$ and ERCC1 in advanced colorectal cancer patients treated with first-line oxaliplatin/fluoropyrimidine. Oncology 72: $364-370$

Okazaki T, Jiao L, Chang P, Evans DB, Abbruzzese JL, Li D (2008) Single-nucleotide polymorphisms of DNA damage response genes are associated with overall survival in patients with pancreatic cancer. Clin Cancer Res 14: 2042-2048

Park DJ, Stoehlmacher J, Zhang W, Tsao-Wei DD, Groshen S, Lenz HJ (2001) A Xeroderma pigmentosum group D gene polymorphism predicts clinical outcome to platinum-based chemotherapy in patients with advanced colorectal cancer. Cancer Res 61: 8654-8658

Park DJ, Zhang W, Stoehlmacher J, Tsao-Wei D, Groshen S, Gil J, Yun J, Sones E, Mallik N, Lenz HJ (2003) ERCC1 gene polymorphism as a predictor for clinical outcome in advanced colorectal cancer patients treated with platinum-based chemotherapy. Clin Adv Hematol Oncol 1: $162-166$

Punt CJ (2004) New options and old dilemmas in the treatment of patients with advanced colorectal cancer. Ann Oncol 15: 1453-1459

Ruzzo A, Graziano F, Loupakis F, Rulli E, Canestrari E, Santini D, Catalano V, Ficarelli R, Maltese P, Bisonni R, Masi G, Schiavon G, Giordani P, Giustini L, Falcone A, Tonini G, Silva R, Mattioli R, Floriani I, Magnani M (2007) Pharmacogenetic profiling in patients with advanced colorectal cancer treated with first-line FOLFOX-4 chemotherapy. J Clin Oncol 25: $1247-1254$

Saldivar JS, Lu KH, Liang D, Gu J, Huang M, Vlastos AT, Follen M, Wu X (2007) Moving toward individualized therapy based on NER polymorphisms that predict platinum sensitivity in ovarian cancer patients. Gynecol Oncol 107: S223-S229

Schrauder M, Frank S, Strissel PL, Lux MP, Bani MR, Rauh C, Sieber CC, Heusinger K, Hartmann A, Schulz-Wendtland R, Strick R, Beckmann MW, Fasching PA (2008) Single nucleotide polymorphism D1853N of the ATM gene may alter the risk for breast cancer. J Cancer Res Clin Oncol 134: $873-882$

Stoehlmacher J, Park DJ, Zhang W, Yang D, Groshen S, Zahedy S, Lenz HJ (2004) A multivariate analysis of genomic polymorphisms: prediction of clinical outcome to 5-FU/oxaliplatin combination chemotherapy in refractory colorectal cancer. Br J Cancer 91: 344-354

Su D, Ma S, Liu P, Jiang Z, Lv W, Zhang Y, Deng Q, Smith S, Yu H (2007) Genetic polymorphisms and treatment response in advanced non-small cell lung cancer. Lung Cancer 56: 281-288

Suk R, Gurubhagavatula S, Park S, Zhou W, Su L, Lynch TJ, Wain JC, Neuberg D, Liu G, Christiani DC (2005) Polymorphisms in ERCC1 and grade 3 or 4 toxicity in non-small cell lung cancer patients. Clin Cancer Res 11: $1534-1538$

Therasse P, Arbuck SG, Eisenhauer EA, Wanders J, Kaplan RS, Rubinstein L, Verweij J, Van GM, van Oosterom AT, Christian MC, Gwyther SG (2000) New guidelines to evaluate the response to treatment in solid tumors. European Organization for Research and Treatment of Cancer, National Cancer Institute of the United States, National Cancer Institute of Canada. J Natl Cancer Inst 92: 205-216

Van Cutsem E, Twelves C, Cassidy J, Allman D, Bajetta E, Boyer M, Bugat R, Findlay M, Frings S, Jahn M, McKendrick J, Osterwalder B, Perez-Manga G, Rosso R, Rougier P, Schmiegel WH, Seitz JF, Thompson P, Vieitez JM, Weitzel C, Harper P (2001) Oral capecitabine compared with intravenous fluorouracil plus leucovorin in patients with metastatic colorectal cancer: results of a large phase III study. J Clin Oncol 19: 4097-4106

Van der Straaten T, Kweekel D, Tiller M, Bogaartz J, Guchelaar HJ (2006) Multiplex pyrosequencing of two polymorphisms in DNA repair gene XRCC1. J Mol Diagn 8: 444-448

Viguier J, Boige V, Miquel C, Pocard M, Giraudeau B, Sabourin JC, Ducreux M, Sarasin A, Praz F (2005) ERCC1 codon 118 polymorphism is a predictive factor for the tumor response to oxaliplatin/5-fluorouracil combination chemotherapy in patients with advanced colorectal cancer. Clin Cancer Res 11: 6212-6217

Wang K, Li M, Bucan M (2007) Pathway-based approaches for analysis of genomewide association studies. Am J Hum Genet 81: 1278-1283

Zhou W, Gurubhagavatula S, Liu G, Park S, Neuberg DS, Wain JC, Lynch TJ, Su L, Christiani DC (2004) Excision repair cross-complementation group 1 polymorphism predicts overall survival in advanced non-small cell lung cancer patients treated with platinum-based chemotherapy. Clin Cancer Res 10: 4939-4943 